\title{
Einheitskasse: Und der Schweizer Souverän sagt wieder nein
}

\section{WILLY OGGIER}

Dr. Willy Oggier ist Inhaber der Firma Willy Oggier Gesundheitsökonomische Beratungen AG und zählt zu den führenden Gesundheitsökonomen der Schweiz. Darüber hinaus ist er ein ausgewiesener Kenner des Gesundheitswesens in Deutschland

\author{
Ende September 2014 hat der Schweizer Souverän \\ erneut dem Anliegen einer Einheitskasse eine Abfuhr \\ erteilt. Im folgenden Beitrag wird die Geschichte \\ dieses immer wieder aufgekommenen Anliegens \\ erläutert und den Gründen nachgegangen, warum \\ diese Initiative letztlich doch erfolglos geblieben ist.
}

\section{Grundzüge des schweizerischen Gesundheitswesens und wichtige Unterschiede zu Deutschland}

Das schweizerische Gesundheitswesen unterscheidet für den Krankheitsfall zwischen einer Grundversicherung, welche ihre gesetzlichen Grundlagen im eidgenössischen Krankenversicherungsgesetz (KVG) hat, und privaten Zusatzversicherungen, welche dem Gesetz über den Versicherungsvertrag (VVG) unterstellt sind. Im Gegensatz zu anderen Ländern sind Zusatzversicherungen in der Schweiz komplementärer und nicht ersetzender Natur. Personen, welche eine Zusatzversicherung abschliessen, können sich demnach nicht der Bezahlung der Grundversicherungsprämie entziehen. Anders als in Deutschland kann man sich also nicht ab einer bestimmten Einkommensgrenze von der gesetzlichen Krankenversicherung und damit von der Solidargemeinschaft verabschieden, indem man zu einer privaten Krankenversicherung wechselt. Auch Beamte haben - mit Ausnahme gewisser gerade aktiv Dienst leistender Militärs - eine Grundversicherungsprämie zu entrichten.

Grund- und Zusatzversicherungen sind mit der Einführung des KVG am 1. Januar 1996 vom Gesetzgeber juristisch getrennt worden. Einige wesentliche Unterschiede lassen sich wie in Tabelle 1 beschrieben darstellen.

Auf der Versicherer-Seite sind im Vergleich zum deutschen System mindestens folgende weiteren wichtigen Unterscheidungs-Elemente zu nennen:

- Die Prämien sind in der Schweiz nicht nur nach Krankenversicherern, sondern auch kantonal und teilweise innerhalb der Kantone auch regional unterschiedlich ausgestaltet. In Deutschland bestanden bereits vor Einführung des Gesundheitsfonds im Bereich der gesetzlichen Krankenversicherung beim gleichen Krankenversicherer weitgehend bundeseinheitliche Prämien.

- Die Prämien in der Krankenversicherung basieren in der Schweiz auf Kopfprämien, welche für Leute in wirtschaftlich bescheidenen Verhältnissen subventioniert werden. In Deutschland kommen Arbeitgeber- und Arbeitnehmerbeiträge zur Anwendung.

- Der Risikoausgleich unter den Krankenversicherern basiert in der Schweiz auf den Kriterien Alter, Geschlecht und Region. Ab dem 1. Januar 2012 wurde zudem berücksichtigt, ob ein Versicherter einen Spital- oder Pflegeheimaufenthalt von länger als drei Nächten aufgewiesen hat. In Deutschland existiert ein morbiditätsorientierter Risikostrukturausgleich, der in der Schweiz vom Parlament als Reaktion auf die letzte Einheitskassen-Volksinitiative beschlossen wurde und erst kurz vor der Einführung steht.

- Die Eigenbeteiligung im schweizerischen Gesundheitswesen ist deutlich höher als in Deutschland. Dies ist u.a. darauf zurückzuführen, dass der 
Leistungskatalog der Grundversicherung in der Schweiz nicht den gleich grossen Umfang wie in Deutschland annimmt und insbesondere im Bereich der Langzeitpflege und der Zahnmedizin mehr direkt aus der eigenen $\mathrm{Ta}$ sche bezahlt werden muss.

- Die Krankenversicherer gelten mit der neuen DRG-Finanzierung in der Schweiz auch die Investitionskostenanteile $a b$, in Deutschland nur die Betriebskosten.

- Die Krankenversicherer werden nach einer Übergangsphase bei sogenannten Listen-Spitälern in der Regel höchstens 45 Prozent der zur Anwendung kommenden DRG zu bezahlen haben (vorbehältlich der Kostenbeteiligung des Patienten), in Deutschland bezahlt der Krankenversicherer die gesamte DRG.

\section{Historischer Rückblick auf bisherige Diskussionen im Zusammenhang mit den Forderungen der Volksinitiative}

Die oben dargestellte Unterscheidung von Grund- und Zusatzversicherungen basiert auf der vom Schweizer Souverän angenommenen Vorlage vom 4. Dezember 1994. Ausgehend von dieser gab es in den letzten Jahren verschiedentlich Versuche, eine Einheitskasse einzuführen und das Wettbewerbssystem unter den

\section{Es gab in den letzten Jahren verschiedentlich Versuche, eine Einheitskasse einzuführen.}

Krankenversicherern in der Grundversicherung in Frage zu stellen. Diese sollen im folgenden dargestellt werden.

\subsection{Abstimmung zum eidgenössi- schen Krankenversicherungsgesetz (KVG) vom 4. Dezember 1994}

In seiner Botschaft an das eidgenössische Parlament vom 6. November 1991 umschreibt der Bundesrat vier Zielsetzungen des neuen Gesetzes: ${ }^{1}$

- Verstärkung der Solidarität

- Kosteneindämmung

- Erweiterung des Leistungskatalogs

- keine fundamentalen Änderungen bei der Finanzierung
Die fehlende Solidarität zwischen Gesunden und Kranken wurde als eines der Hauptprobleme des damals gültigen Rechts bezeichnet. Ausserdem trage dieses der Solidarität zwischen Reichen und Armen nicht genügend Rechnung. Deshalb schlug der Bundesrat im Rahmen des Versicherungsobligatoriums die Einführung der vollen Freizügigkeit der Versicherten und die Erleichterung der über einem gewissen Einkommensanteil liegenden Prämienlast durch individuelle Prämienverbilligungen vor.

Die Eindämmung der Kostensteigerung im Gesundheitswesen wurde als eines der Hauptziele der Revision bezeichnet. Was der Bundesrat darunter verstand, umschrieb er auf folgende Art: ${ }^{2}$ „Das Ziel der in dieser Vorlage vorgeschlagenen Kostendämpfungsmassnahmen wäre dann vollständig erreicht, wenn sich die jährliche Zunahme der Gesundheitsausgaben pro Kopf der Bevölkerung der allgemeinen Lohn- und Preisentwicklung anpasst. Dieses Idealziel wird mittelfristig kaum zu erreichen sein. Hingegen muss es uns gelingen, ein noch stärkeres Auseinanderklaffen von Gesundheitsausgaben und Löhnen zu stoppen, das heisst die Zunahme der Gesundheitsausgaben näher an die Lohnentwicklung heranzubringen. Die Kostendämpfungsmassnahmen werden nach der Einführung des Gesetzes allerdings nicht sofort greifen. Die einzelnen Instrumente entfalten erst im gegenseitigen Zusammenspiel ihre volle Wirkung. Zum Gelingen der Reform beitragen müssen alle Partner im Gesundheitswesen.“

Bezüglich der Ausweitung des Leistungskatalogs äusserte sich die Bundesregierung wie folgt: ${ }^{3}$ „Tatsächlich kann man sich fragen, ob es nicht paradox ist, in ein und demselben Gesetz eine Ausdehnung der Leistungen einerseits und die Schaffung von verschiedenen Instrumenten zur Eindämmung der Kosten andererseits vorzusehen. Diese Bedenken sind zwar verständlich, aber unzutreffend. Die Ausdehnung der Leistungen ist unerlässlich. Damit besteht die Möglichkeit, zum Teil weit zurückliegenden unbestrittenen - Forderungen Rechnung zu tragen (...) und bestimmte stossende Lücken zu beseitigen (z.B. durch Ein- führung der zeitlich unbeschränkten Übernahme von Spitalpflegeleistungen sowie die Vergütung bestimmter Zahnbehandlungen).“

Im Bereich der Finanzierung wurde die Kopfprämie beibehalten. Der Bundesrat schreibt dazu in der Botschaft u.a.: „Die Kopfprämie ist die der heutigen und auch künftig vorgesehenen Organisation der Krankenversicherung entsprechende Art der Prämienerhebung. Wir haben bereits 
Tabelle 1: Wichtige Unterschiede zwischen Grund- und Zusatzversicherungen

Grundversicherung nach KVG

Grundlage sind Gesetz und Verordnungen.

Obligatorium und Aufnahmepflicht durch die Versicherer

Es besteht ein fest definierter gesetzlicher Leistungskatalog mit Einschluss der Mutterschaft.

Finanzierung nach Umlageverfahren (die Ausgaben werden laufend durch Einnahmen - Prämien und Kostenbeteiligung gedeckt)

Einheitsprämien pro Versicherer und Region. Prämienreduktion für Kinder und Jugendliche. Prämienverbilligung.

Die Versicherten können bei normaler (d.h. nicht wählbarer Franchise) und im Rahmen der ordentlichen Grundversicherung (d.h. keine besondere Versicherungsform) den Versicherer mit einer Kündigungsfrist von drei Monaten auf Semesterende ohne Nachteile wechseln. Der Versicherer kann dem Versicherten nicht kündigen. Ein Ausschluss ist kaum möglich.

Keine Vorbehalte bei Versicherungswechsel

Unbeschränkte Versicherungsdauer

Prinzip der Gegenseitigkeit und Gleichbehandlung

Verjährung von Leistungsansprüchen nach fünf Jahren

Keine Kollektivversicherungen
Zusatzversicherung nach VVG

Grundlage ist der Vertrag mit den allgemeinen Versicherungsbedingungen (AVB).

Keine Aufnahmepflicht; Ausnahme: Bisherige Zusatzversicherte nach altem Recht haben beim gleichen Versicherer Anrecht auf einen Vertrag, der die bisherigen Leistungen abdeckt.

Der Versicherer bestimmt, welche ergänzenden Leistungen zur Grundversicherung er anbieten will. Mutterschaft auf Antrag.

Keine Vorschriften. In der Praxis: Bedarfsdeckungs- oder Kapitaldeckungsverfahren oder eine Mischform der beiden (Rückstellung für spätere Verpflichtungen oder Ansparen von Kapital für Risiken im Alter).

Keine Vorschriften für Prämiengestaltung. In der Praxis fördert der Wettbewerb aber risikogerechte Prämien.

Beide Parteien können in einem Versicherungsfall sofort (bis vierzehn Tage nach Zahlung) oder auf Vertragsende mit einer dreimonatigen Kündigungsfrist kündigen (ohne Kündigung stillschweigende Vertragsverlängerung um ein Jahr). Viele Versicherer verzichten einseitig auf ihr Recht, im Versicherungsfall zu kündigen.

Unbeschränkte Vorbehalte oder Leistungsausschlüsse möglich

Die Dauer der Verträge kann limitiert werden.

Prinzip von Treu und Glauben

Verjährung von Leistungsansprüchen nach zwei Jahren

Kollektivversicherungen sind möglich.

Quelle: KSK aktuell, Nr. 9, September 1996, aktualisiert.

Vereinbarungen oder Regelungen enthalten sind: Sondervertragsverbote zu Lasten von Verbandsmitgliedern, Verpflichtung von Verbandsmitgliedern auf bestehende Verbandsverträge, Konkurrenzverbote zu Lasten von Verbandsmitgliedern, Exklusivitätsund Meistbegünstigungsklauseln.

- Die Krankenversicherer können nach Art. 62 Abs. 1 KVG die Prämien für Versicherungen mit eingeschränkter Wahl der Leistungserbringer vermindern. Diese Bestimmung bildet die Basis für die alternativen Versicherungsmodelle und Managed Care in der Schweiz.

\subsection{Abstimmung zur SPS-/ SGB-Lohnprozent-Initiative vom 4. Dezember 1994}

Das System der Kopfprämie wurde am 4. Dezember 1994 in doppelter Weise vom Souverän in der Volksabstimmung bestätigt. Einerseits wurde dem neuen Krankenversicherungsgesetz zugestimmt, anderseits wurde eine Volksinitiative „für eine gesunde Krankenversicherung “ des
Schweizerischen Gewerkschaftsbunds (SGB) und der Sozialdemokratischen Partei der Schweiz (SPS) mit 460.674 Ja- (23.4 Prozent) zu 1.504.177 NeinStimmen (76.6 Prozent) und von allen Ständen verworfen. Die Volksinitiative wies folgenden Wortlaut auf:

\section{Art. 34bis}

1 Der Bund richtet auf dem Wege der Gesetzgebung die Kranken- und die Unfallversicherung ein. Er überträgt deren Durchführung Einrichtungen, die die Versicherung nach dem Grundsatz der Gegenseitigkeit betreiben.

1. Die Unfallversicherung ist für alle Arbeitnehmer obligatorisch. Der Bund kann das Obligatorium auf weitere Kreise der Bevölkerung ausdehnen.

2. Die Krankenpflegeversicherung ist für die ganze Bevölkerung obligatorisch. Sie deckt obne zeitliche Begrenzung die Behandlungskosten bei Krankheit und, soweit nicht anderweitig von Gesetzes wegen versichert, bei Unfall; eingeschlossen sind die Hauskrankenpflege und Leistungen der Gesundheitsvorsorge.
Die Versicherung wird finanziert:

a. durch Beiträge der Versicherten nach Massgabe ihrer wirtschaftlichen Leistungsfähigkeit; bei Erwerbstätigen wird das volle Erwerbseinkommen zur Bemessung des Beitrages herangezogen, wobei die Arbeitgeber bei Arbeitnehmern mindestens die Hälfte übernehmen. Kinder zablen keine Beiträge;

b. durch einen Beitrag des Bundes von mindestens einem Viertel der Ausgaben; das Gesetz regelt die Beteiligung der Kantone am Bundesbeitrag.

Das Gesetz kann eine Beteiligung der Versicherten an den von ibnen verursachten Kosten von höchstens einem Fünftel ibres Beitrages pro Jahr vorseben; keine Kostenbeteiligung darf bei Vorsorgemassnahmen erhoben werden.

3. Die Krankengeldversicherung ist für alle Arbeitnehmer obligatorisch. Sie zahlt bei Lohnausfall infolge Krankheit ein Taggeld von mindestens 80 Prozent des versicherten Lohnes. 
Die Versicherung wird finanziert durch Beiträge in Prozenten des versicherten Lohnes, wobei die Arbeitgeber mindestens die Hälfte tragen. Der Bund sorgt dafür, dass sich gesetzlich nicht versicherte Personen der Taggeldversicherung für Leistungen bei Krankheit oder Unfall anschliessen können.

2 Die Behandlungsfreiheit ist im Rahmen der Wirtschaftlichkeit gewährleistet. Bund und Kantone sorgen für die wirtschaftliche Verwendung der Finanzmittel der Versicherung. Zu diesem Zweck erlassen sie Tarif- und Abrechnungsvorschriften und legen verbindliche Spitalplanungen fest.

\subsection{Abstimmung zur SP-Volksinitiative „Gesundheit muss bezahlbar bleiben (Gesundheitsinitiative)" vom 18. Mai 2003}

Am 18. Mai 2003 lehnte der Souverän die Volksinitiative „Gesundheit muss bezahlbar bleiben" der SPS mit 625.570 Ja- zu 1.681.943 Nein-Stimmen für viele politische Beobachter überraschend deutlich ab. Sämtliche Kantone lehnten die Initiative $a b$ und 72.9 Prozent der Stimmenden. Gemäss der Vox-Befragung stimmten selbst SPSympathisanten nur zu 59 Prozent für die Initiative ${ }^{5}$.

Gemäss Initiative hätte die Bundesverfassung wie folgt geändert werden sollen (die ebenfalls enthaltenen Übergangsbestimmungen werden aus Gründen der Lesbarkeit hier nicht dargestellt):

Art. 34bis

1. Der Bund erlässt Vorschriften über die Kranken-und die Unfallversicherung.

2. Die obligatorische Krankenversicherung erfolgt durch gemeinnützige Krankenversicherer. Sie garantiert allen Versicherten eine qualitativ bochstehende, bedarfsgerechte und kostengünstige medizinische Versorgung.

3. Die obligatorische Krankenversicherung wird insbesondere finanziert aus:

a. zusätzlichen, zweckgebundenen Einnabmen aus der Mehrwertsteuer in gesetzlich festgelegtem Umfang. b. in mindestens gleich hohem Umfang durch Beiträge der Versicherten; diese Beiträge werden im Verhältnis zum Einkommen und zum realen Vermögen sowie unter Berücksichtigung der Familienlasten festgelegt.

4. Die Krankenversicherer erhalten pro versicherte Person Beiträge aus den unter Abs. 3 genannten Mitteln. Dabei werden die unterschiedlichen Risiken der Versicherer ausgeglichen. Überschüsse werden den Versicherten zurückerstattet.

5. Bund und Kantone sorgen für eine wirksame Kostendämpfung im Gesundheitswesen.

Der Bund trifft dazu insbesondere folgende Massnabmen:

a. Er regelt die Spitzenmedizin und koordiniert die Gesundheitsplanungen der Kantone.

b. Er bestimmt die Maximalpreise der in der obligatorischen Krankenversicherung erbrachten Leistungen unter Einschluss der Medikamente.

c. Er erlässt Zulassungsbestimmungen für die Leistungserbringer und sorgt für eine wirksame Qualitätskontrolle.

d. Werden übermässige Leistungsmengen erbracht, ergreift er nach Sparten und Regionen differenziert weitere Kostendämpfungsmassnahmen.

Die Kantone können im Bereich der Gesundheitsplanung weitergehende Massnabmen treffen.

\section{In den vergangenen Jahren} hatten parlamentarische Vorstösse und Initiativen zum Thema Einheitskasse keinen Erfolg.

\subsection{Parlamentarische Vorstösse zum Thema Einheitskasse}

Darüber hinaus gab es in den vergangenen Jahren u.a. folgende gescheiterte parlamentarische Vorstösse und Initiativen zum Thema Einheitskasse auf der Ebene Bund (in den beiden Parlamentskammern National- bzw. Ständerat) und Kantone:

- Der Nationalrat lehnt im Juni 1999 die parlamentarische Initiative Fasel für die Schaffung einer schweizeri- schen Einheitskasse mit 91 gegen 64 Stimmen ab.

- Das Genfer Kantonsparlament verwirft im Mai 2002 einen Vorstoss der Linksallianz zur Einführung einer kantonalen Krankenkasse mit 43 gegen 35 Stimmen.

- Einen ähnlich lautenden Vorstoss lehnt das Freiburger Kantonsparlament im Juni 2002 ab.

- Die Konferenz der Westschweizer Sanitäts- und Sozialdirektoren (CRASS) verzichtet im Herbst 2003 darauf, ein Projekt zur Einführung einer regionalen Einheitskasse in der Westschweiz weiter zu verfolgen. Sie lässt aber einen Bericht zur Frage einer schweizerischen Krankenkasse ausarbeiten, welcher im Dezember 2003 veröffentlicht wird.

- Nationalrat und Ständerat geben im März bzw. Juni 2003 der parlamentarischen Initiative Zysiadis zur Schaffung einer nationalen Einheitskasse keine Folge.

- Ständerat und Nationalrat sprechen sich im März bzw. Juni 2003 gegen eine Standesinitiative des Kantons Jura „für eine zentrale Landeskasse und einkommensabhängige Prämien“ aus.

- Der Ständerat gibt im Dezember 2003 einer Initiative des Kantons Tessin für die Einführung einer Krankenkasse auf Bundesebene keine Folge.

\subsection{Die Initiative des Mouvement populaire des familles}

Am 11. März 2007 wurde die Volksinitiative „Für eine soziale Einheitskrankenkasse“ mit 641.917 Ja- (28.8 Prozent) zu 1.590.213 Nein-Stimmen (71.2 Prozent) abgelehnt. Sie hatte folgenden Wortlaut (ohne Übergangsbestimmungen):

Art. 117 Abs. 3 KVG (neu):

Der Bund richtet eine Einheitskasse für die obligatorische Krankenpflegeversicherung ein. Im Verwaltungsrat und im Aufsichtsrat sind die Behörden, die Leistungserbringer und die Interessenvertretung der Versicherten mit jeweils gleich vielen Personen vertreten. Das Gesetz regelt die Finanzierung der Kasse. Es legt die Prämien nach der wirtschaftlichen Leistungsfähigkeit der Versicherten fest.

5 Neue Zürcher Zeitung, Nr. 165, 19./ 20. Juli 2003. 


\subsection{Volksinitiative „Für eine öffentliche Krankenkasse“}

Am 28. September 2014 lehnte der Schweizer Souverän die Volksinitiative „Für eine öffentliche Krankenkasse“ mit 932.761 Ja- (38.2 Prozent) zu 1.512.116 Nein-Stimmen (61.8 Prozent) ab. Im Gegensatz zu den vorherigen Volksinitiativen war dieses Mal das Anliegen der Einheitskasse nicht mit einer Änderung der Prämienfinanzierung verbunden. Die Initiative hatte folgenden Wortlaut (ohne Übergangsbestimmungen):

Art. 117 Abs. 3 KVG (neu) und 4 (neu): 3 Die soziale Krankenversicherung wird von einer einheitlichen nationalen öffentlich-rechtlichen Einrichtung durchgeführt. Deren Organe werden namentlich aus Vertreterinnen und Vertretern des Bundes, der Kantone, der Versicherten und der Leistungserbringer gebildet.

4 Die nationale Einrichtung verfügt über kantonale oder interkantonale Agenturen. Diese legen namentlich die Prämien fest, ziehen sie ein und vergüten die Leistungen. Für jeden Kanton wird eine einheitliche Prämie festgelegt, diese wird aufgrund der Kosten der sozialen Krankenversicherung berechnet.

\subsection{Fazit}

Das Anliegen der Einführung einer Einheitskasse erweist sich auf schweizerischer Ebene nicht als neu. Auch wenn in der Geschichte der Sozialversicherungen in der Schweiz nicht selten mehrere Anläufe notwendig waren, um zu Reformen zu kommen, erstaunt insbesondere beim Anliegen der Einheitskasse der geringe zeitliche Abstand, mit dem diese Forderung immer wieder - sei es direkt oder indirekt über die Forderung nach Prämien gemäss der wirtschaftlichen Leistungsfähigkeit - auf das politische Parkett gebracht wird.

Dass dieses Anliegen auch bei wiederholten Versuchen den Durchbruch nicht schaffte, dürfte einerseits damit zu tun haben, dass Regierung und Parlament insbesondere mit der Einführung des Krankenversicherungsgesetzes vor rund 20 Jahren und mit der neuesten Reform zur Verbesserung des Risikoausgleichs in Richtung Morbidi- tätsorientierung dem Anliegen Wind aus den Segeln nehmen konnten.

Darüber hinaus gibt es auch weitere Argumente, die gegen die Radikallösung Einheitskasse sprechen, denen im Folgenden zum Abschluss nachgegangen werden soll.

\section{Argumente gegen die Einheitskasse}

Einer der grössten Vorteile für die Versicherten bei einem wettbewerblichen System der Krankenversicherer in einer sozialen Krankenversicherung mit Aufnahmezwang besteht darin, dass die Versicherten über Wechselfreiheit verfügen. Wechselfreiheit führt in der Regel zu mindestens folgenden Vorteilen:

- Der Versicherte kann bei gleichem Leistungskatalog einen Krankenversicherer mit einer günstigeren Prämie wählen und damit sein individuelles verfügbares Einkommen erhöhen.

- Der Versicherte kann bei gleichem Leistungskatalog den Versicherer wechseln, wenn er mit seinem Service nicht zufrieden ist.

- Der Versicherte kann bei gleichem Leistungskatalog den Versicherer wechseln, wenn er das Gefühl hat, er werde wegen eines Leidens oder einer Krankheit schlecht(er) behandelt.

Bei Einführung einer Einheitskasse ist davon auszugehen, dass die kostendämpfenden Anreize spätestens mittel- bis langfristig weitgehend entfallen. Denn Monopolisten verspüren in der Regel keinen Anreiz, die Preise zu senken. Monopolsituationen weisen zudem eine hohe Wahrscheinlichkeit auf, dass sowohl die Servicequalität als auch die Produkte-Vielfalt beeinträchtigt werden.

Wenn die Kantone auch noch in der Trägerorganisation vertreten sein sollen, führt dies zu einer weiteren Verflechtung statt Entflechtung ihrer Rollen. Kantone könnten dann versucht sein, über die Einheitskasse ihren eigenen Spitälern bevorzugte (sprich: höhere) Entgelte zukommen zu lassen. Benachteiligungen von Privatkliniken und frei praktizierenden Leistungserbringern wären die Folge.

Die Einführung einer Einheitskasse dürfte zu erheblichen Wettbewerbsverzerrungen im Rahmen der Zusatzversicherungen führen, weil jene Versicherung, welche als Einheitskasse waltet, gegenüber allen anderen Versicherern im Bereich der Zusatzversicherungen über einen Informationsvorsprung verfügt. Darf die Einheitskasse im Bereich der Zusatzversicherungen nicht tätig werden, ergibt sich wiederum das Problem, dass viele zusatzversicherte Personen Grund-
Eine längerfristige Abflachung des Prämienanstiegs dürfte nur dann zu erreichen sein, wenn auf der leistungserbringerseite angesetzt wird. Denn dort sind die Gründe zu suchen.

und Zusatzversicherungen im Gegensatz zu heute bei unterschiedlichen Versicherern haben müssen.

Wäre die Einheitskassen-Initiative angenommen worden, hätten die Krankenversicherer wohl kein oder kaum Interesse mehr an der Aufrechterhaltung einer seriösen Reserven- und Rückstellungspolitik gehabt. Damit besteht das Risiko, dass im Zeitpunkt der Übertragung von Aktiva und Passiva der heutigen Versicherer auf die Einheitskasse kaum oder gar keine Reserven und Rückstellungen mehr vorhanden sind.

Eine längerfristige Abflachung des Prämienanstiegs dürfte nur dann zu erreichen sein, wenn dort angesetzt wird, wo die Gründe für die Anstiege zu suchen sind. Dies ist in erster Linie auf der Leistungserbringerseite und nicht bei den Verwaltungskosten, welche rund 5 Prozent der gesamten KrankenversichererAusgaben ausmachen und auf die sich die Hauptargumente der EinheitskassenBefürworter richteten. 